Research and Clinical Studies in Headache Vol. 4. Pathophysiologic, Diagnostic and Therapeutic Aspects of Headache

Volume editors: Mary E. Granger and G. Poch. Pp. vii +128 , hard cover, illustrated. S. Karger AG: Basel, 1976. DM 62; US \$24.00.

This attractively presented book is the fourth in a series of volumes dealing with pain in the head. It consists of nine chapters by different authors touching on a wide number of topics in the headache field. The description on the jacket states that this volume provides an excellent balance between the experimental and clinical aspects of the headache problem. Unfortunately, this balance is hard to convey in a number of uncoordinated chapters and the newcomer to the headache world may well find it confusing and fail to discern the relative significance of the subjects under consideration.

In presenting their information, several of the authors assume a basic knowledge on the part of the reader which will make the book of considerably greater interest to those who are already familiar with the field. For example, the chapter on 'The Effects of Humoral Agents and Antimigraine Drugs on the Cranial Circulation of the Monkey' is written for the reader who is well versed in the anatomy and physiology of the cerebral circulation.

There are informative chapters on radiological techniques in the examination of patients with headaches, and on headaches and head pains associated with diseases of the eye. There are interesting though brief chapters on thermography, the rheoencephalogram and the electroencephalogram. The book, however, provides little practical guidance to the physician faced with a headache sufferer, although it may well draw his attention to some of the many fascinating aspects of the headache problem. It seems a pity that these various aspects of headache have not been grouped in co- $z$ ordinated sections in the volumes that make up this series, as $\mathbb{D}$ it is difficult to assess the relevance of some of the contributions when presented in isolation.

Total Knee Replacement (Proceedings of a Conference) Pp. 192, illustrated, hard cover. Mechanical Engineering Publications Ltd for The Institution of Mechanical $\frac{O}{\bar{O}}$ Engineers: London and New York, 1976. £15.00.

This is a collection of the papers delivered at a symposium $\stackrel{\nabla}{\mathbb{D}}$ arranged by the Institution of Mechanical Engineers and the British Orthopaedic Association which took place in September 1974. The choice of subject for a meeting between experts $\vec{\nabla}$ from two major disciplines was excellent. Arthroplasty of the knee is a problem of great topical interest. Whereas hip $\overrightarrow{\vec{\omega}}$ replacement is accepted as a standard procedure with, on the whole, uniformly good results, knee replacement is still in an early stage of development. This is reflected in the manye varieties of artificial knee joints which are available. These 3 . ranged from hinged prostheses to those with two separate if components made of metal and polyethylene, and included $N$ the compromise of the constrained two-part device favoured $\rightarrow$ by both Attenborough and Deane. Perspective is achieved by $A$ discussion of the engineering considerations and the bio- $\infty$ mechanics of the knee. The report by Arden and Kamdar on N the complications of arthroplasty of the knee is somewhat chastening and should curb the enthusiasm of the surgeon. Although the material which makes up the book is now 2. years old, this volume serves a useful function. A large amount of well documented information has been collected $\mathbb{D}$ about a rapidly developing subject. It is of interest to all orthopaedic surgeons dealing with degenerative arthritis.

\title{
ANNOUNCEMENT
}

\section{The 1977-1978 Johananoff International Fellowship for Advanced Biomedical Studies}

The Johananoff Fellowship is offering a foreign (non-Italian) distinguished scientist, internationally renowned for outstanding contributions in cancer chemotherapy and/or immunology, cardiovascular pharmacology, neuropsychopharmacology, or drug metabolism, the opportunity to spend his/her sabbatical year at the Mario Negri Institute for Pharmacological Research in Milan, reading, thinking and writing, producing an 'Advanced Study' on a chosen topic of his/her specific competence.

Candidates should be other than Italian citizens. Only scientists from academic institutions, non-profit organizations or governmental agencies will be considered. Preference shall be given to mid-career scientists, belonging to the age group 35 to 50 .

The Award is in the amount of $\$ 15,000$ U.S. for 1 year, payable in Milan, in monthly instalments. There are no objections to the Johananoff Fellow receiving his regular stipend or any other support.

Applications, submitted according to the specific instructions, should be received by 31 January 1977 and must include a curriculum vitae, list of publications and outline of the proposed studies.

The award will be announced by 30 March 1977. The recipient can come to Milan any date thereafter, provided the arrival date falls within 1977.

Requests for further information and application kits should be airmailed to:

The Johananoff Fellowship Committee Istituto di Ricerche Farmacologiche 'Mario Negri' Via Eritrea, 62

20157 Milan, Italy

Tel. (02) 355.4546; Telex 37268 NEGRINST. 